\title{
Hypermethylation of the RSK4 promoter associated with $B R A F$ V600E promotes papillary thyroid carcinoma
}

\author{
YANAN YIN ${ }^{1}$, KUI $\mathrm{CHE}^{2}$, JIANXIA HU ${ }^{2}, \mathrm{HUI} \mathrm{HUA}^{3}, \mathrm{ANBING} \mathrm{DONG}^{3}$, \\ JUERU WANG $^{3}$, JIARUI YU ${ }^{1}$, QING ZHANG ${ }^{1}$, SHIHUA ZHAO $^{1}$, YUHANG ZHAO ${ }^{1}$, \\ PING WANG $^{1}$, FEI WANG ${ }^{1}$, YANGANG WANG ${ }^{1}$, JINGWEI CHI $^{2}$ and WENHAI SUN ${ }^{3}$ \\ ${ }^{1}$ Department of Endocrinology and Metabolism, ${ }^{2}$ Qingdao Key Laboratory of Thyroid Diseases; \\ ${ }^{3}$ Department of Thyroid Surgery, The Affiliated Hospital of Qingdao University, Qingdao, Shandong 266000, P.R. China
}

Received November 25, 2019; Accepted February 6, 2020

DOI: 10.3892/ijo.2020.4999

\begin{abstract}
Ribosomal S6 kinase 4 (RSK4) is a putative tumor suppressor gene which is inactivated by epigenetic events in a number human malignancies; however, its role in papillary thyroid carcinoma (PTC) remains largely unknown. The aim of this study was to explore the methylation status of the RSK4 promoter in PTC, and to determine its potential role in thyroid carcinogenesis. Reverse transcription-quantitative PCR (RT-qPCR) and western blot analyses were performed to examine the RSK4 mRNA and protein levels, respectively. Methylation-specific PCR (MSP) and bisulfite genomic sequencing (BGS) were used to analyze methylation status of the $R S K 4$ gene. Sanger sequencing was further carried out to detect the BRAF V600E mutation. Cell proliferation assay was finally performed to evaluate the role of hypermethylation in the growth of PTC cells. The association between RSK4 methylation and the clinicopathological characteristics of patients with PTC was assessed. The methylation frequency of the RSK4 promoter in PTC tissues was higher than that in paired paracancerous tissues. Coincidentally, the RSK 4 mRNA levels were also downregulated in PTC tissues when compared with the paracancerous counterparts. The hypermethylation of $R S K 4$ was associated with tumor size and lymph node metastasis. Furthermore, the BRAF V600E mutation may influence RSK4 expression and methylation. Moreover, RSK4 hypermethylation was observed in thyroid cancer cell lines, which was consistent with a lack of RSK4 expression. Upon the
\end{abstract}

Correspondence to: Dr Wenhai Sun, Department of Thyroid Surgery, The Affiliated Hospital of Qingdao University, 16 Jiangsu Road, Shinan, Qingdao, Shandong 266000, P.R. China

E-mail: sunwenhaiqyfy@163.com

Dr Jingwei Chi, Qingdao Key Laboratory of Thyroid Diseases, The Affiliated Hospital of Qingdao University, No. 16 Jiangsu Road, Shinan, Qingdao, Shandong 266000, P.R. China

E-mail: cjw_03@126.com

Key words: papillary thyroid carcinoma, ribosomal S6 kinase 4, methylation, BRAF V600E
5-Aza-deoxycytidine treatment of thyroid cancer cells, RSK4 expression was significantly upregulated, while cell proliferation was inhibited. On the whole, the findings of the present study demonstrate that the hypermethylation of the RSK4 promoter may be one of the mechanisms responsible for the poor RSK4 expression in PTC. Thus, these data suggest that $R S K 4$ may serve as a molecular target for the early diagnosis and treatment of PTC.

\section{Introduction}

In recent decades, thyroid cancer has become a very common type of endocrine malignancy whose incidence has rapidly increased worldwide (1). As the most frequent histological type of thyroid cancer, the increased incidence of papillary thyroid carcinoma (PTC) has been pivotal to the increased numbers of patients affected by thyroid cancer (2). More sensitive reliable methods of diagnostic imaging and surveillance have also contributed to the higher detection of PTC (3). Despite the low mortality rate due to PTC, the disease recurrence is high (4). Therefore, exploring the potential molecular mechanisms responsible for carcinogenesis and the development of PTC is imperative.

Similar to other malignancies, thyroid cancer is a complex disease intertwined by multiple genetic and environmental factors (5). Recently, the reduced expression of ribosomal S6 kinase 4 (RSK4) has been implicated in the carcinogenesis of colon, kidney, breast, ovarian and endometrial cancers, suggesting a role as a putative tumor suppressor gene (6-10). RSK4 belongs to the 90-kDa S6 ribosomal kinase family, and its coding gene is located on the $\mathrm{X}$-chromosome (Xq21.1) (9). RSK4 mediates signal transduction downstream of the mitogen-activated protein kinase (MAPK) cascades, serving as a serine/threonine kinase (11). Due to this kinase activity, RSK4 also plays a significant role in the regulation of cellular survival, division and differentiation (9). It has been reported that RSK4 overexpression is capable of inducing a senescence-like phenotype in vitro, while its downregulation can induce the escape from cellular senescence and, consequently, immortalization during the early stages of tumorigenesis (7). PTC is driven by multiple genetic elements, in which the BRAF V600E mutation is the most common 
oncogenic point mutation, occurring with a prevalence of $45 \%$ in PTC (12). BRAF V600E mutation aberrantly activates the MAPK pathway, and plays a crucial role in the carcinogenesis of thyroid cancer (13-15). Furthermore, RSK4 is likely to act directly on or downstream of ERK, therefore inhibiting the MAPK signaling cascade (16).

Epigenetics can be described as a heritable modification of gene function that does not affect the genomic DNA sequence, although ultimately, it leads to a change in phenotype. In the mammalian genome, the methylation of gene promoters is one of the most common epigenetic events (17). As a common feature of tumorigenesis, hypermethylation can block the activity of certain genes which function as tumor suppressors, while demethylation can induce gene reactivation and expression (18). Therefore, the inactivation of gene transcription by promoter methylation may serve as a potential platform for the early diagnosis, risk and prognosis assessment, treatment and management of cancers (19).

The implication of $R S K 4$ methylation in human PTC has not been previously reported, at least to the best of our knowledge. Thus, the present study demonstrates that RSK4 hypermethylation partly contributes to the downregulation of RSK4 expression and is related to the tumor size and lymph node metastasis in PTC. Moreover, the present study investigated the association between RSK4 and the BRAF V600E mutation in PTC. The present study indicates that $R S K 4$ potentially functions as a tumor suppressor and also participates in the carcinogenesis of PTC.

\section{Materials and methods}

Patients and tissue samples. A total of 226 paired papillary thyroid carcinoma tissues and corresponding paracancerous counterparts were acquired between January, 2018 and February, 2019, after obtaining informed consents, from patients at the Affiliated Hospital of Qingdao University (Qingdao, China). For patients $<18$ years old, their written informed consents were provided by their parents. Tissue samples were collected, immediately frozen in liquid nitrogen and stored at $-80^{\circ} \mathrm{C}$ until further analysis. All samples were confirmed by pathological assessment. In this study population, 169 patients were female and 57 were male. The median age at diagnosis was 45 years (range, 17-76 years). Patients with primary PTC were included and those who had received chemotherapy or radiotherapy prior to surgery were excluded. The study design and implementation were approved by the Ethics Committee of the Affiliated Hospital of Qingdao University.

Cells and cell culture. Immortalized thyroid cancer cell lines (TPC-1 and BHT101) and a normal thyroid epithelial cell line (Nthy-ori3-1) were obtained from ExPASy Bioinformatics Resource Portal and Type Culture Collection of the Chinese Academy of Sciences, respectively. TPC-1 and BHT101 cells were cultured in DMEM supplemented with 10\% FBS (both from Gibco; Thermo Fisher Scientific, Inc.). Nthy-ori3-1 cells were cultured in F12K medium (Gibco; Thermo Fisher Scientific, Inc.) supplemented with $10 \%$ FBS. The cells were maintained in incubator at $37^{\circ} \mathrm{C}$ and $5 \% \mathrm{CO}_{2}$. When required, the TPC-1, BHT101 and Nthy-ori3-1 cells were treated with
$10 \mu \mathrm{M}$ 5-Aza-deoxycytidine (5-Aza; Sigma-Aldrich; Merck $\mathrm{KGaA}$ ) for 5 consecutive days.

Reverse transcription-quantitative $R C R$ ( $R T$-qPCR). Total RNA was extracted using TRIzol reagent (T9424, Sigma-Aldrich; Merck KGaA) according to the manufacturer's protocol. Total RNA (3 $\mu \mathrm{g})$ was reverse transcribed at $37^{\circ} \mathrm{C}$ for $15 \mathrm{~min}$ and $85^{\circ} \mathrm{C}$ for $5 \mathrm{sec}$ using the PrimeScript ${ }^{\mathrm{TM}} \mathrm{RT}$ reagent kit with gDNA Eraser (Code no. RR047, Takara Bio, Inc.). Subsequently, single-stranded cDNAs were subjected to qPCR using TB Green ${ }^{\circledR}$ Premix Ex Taq ${ }^{\mathrm{TM}}$ (Code no. RR420, Takara Bio, Inc.) on a LightCycler ${ }^{\circledR} 96$ Real-Time PCR System (Cobas z480, Roche). The amplification conditions were as follows: $95^{\circ} \mathrm{C}$ for $30 \mathrm{sec}$, followed by 30 cycles of $95^{\circ} \mathrm{C}$ for $5 \mathrm{sec}$ and $60^{\circ} \mathrm{C}$ for $30 \mathrm{sec}$. The following gene-specific qPCR primers were used in this study: RSK4 forward, 5'-GAACAG ACATTTCCAGCTAAAAAGG-3' and reverse, 5'-TGCCTG AAGATCCAGCAACTAA-3'; BRAF forward, 5'-TGGGGA ACGGAACTGATTTTTC-3' and reverse, 5'-TTTTGTGGT GACTTGGGGTTG-3'. GAPDH was used as a reference gene (normalizer) and amplified using the following primers: Forward, 5'-TGCACCACCAACTGCTTAGC-3' and reverse, 5'-GGCATGGACTGTGGTCATGAG-3'. The relative mRNA expression of $R S K 4$ was analyzed using the $2^{-\Delta \Delta C q}$ method (20).

Western blot analysis. Cell samples were washed twice with PBS and then lysed in Laemmli buffer (2\% SDS, $10 \%$ glycerol, 0.125M Tris-Cl, pH 6.8). The BCA method was used for protein quantification. The concentrations of separating gel and stacking gel were 10 and 5\%. Equal amounts of total protein $(20 \mu \mathrm{g})$ were resolved by SDS-PAGE and transferred onto nitrocellulose membranes. The membranes were blocked with $5 \%$ skim milk and incubated with respective primary antibodies overnight at $4^{\circ} \mathrm{C}$. The membranes were washed 3 times with TBST buffer (containing 0.1\% Tween-20) and then incubated with corresponding HRP-conjugated secondary antibody for $1 \mathrm{~h}$ at room temperature. The membranes were washed again, 3 times with TBST, prior to protein detection. Specific protein bands were developed by chemiluminescence using a commercial ECL kit (Millipore). The following antibodies were used for western blot analysis: Mouse anti-RSK4 monoclonal antibody (1:500, 27330, Novus Biologicals); rabbit anti-GAPDH polyclonal antibody (1:2,000, E-AB-20059, Elabscience Biotechnology); the secondary antibodies were as follows: Goat anti-rabbit/goat anti-mouse $\mathrm{IgG}$ polyclonal antibody (1:10,000, 111-035-003/115-005-003, Jackson ImmunoResearch Laboratories).

DNA extraction and sodium bisulfite modification. Total genomic DNA was extracted from the tissues and cells using a Genomic DNA extraction kit (Aidlab). All procedures followed according to manufacturer's protocol. Genomic DNA samples (3 $\mu \mathrm{g}$ each) was bisulfite-modified and then purified according to the manufacturer's protocol. (Qiagen).

Methylation-specific PCR (MSP). In total, 134 pairs of specimens of PTC patients were included. Specific primers of RSK4 for the unmethylated (5'-TTTTTATTGATGTTTGGG TGAT-3' and 5'-AACAACAACCACTCAATAATAAC-3') and methylated reactions (5'-TTATTGACGTTTGGGTGAC-3' 
A

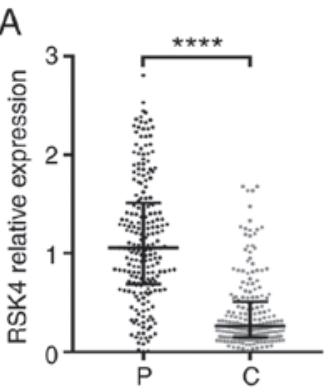

C

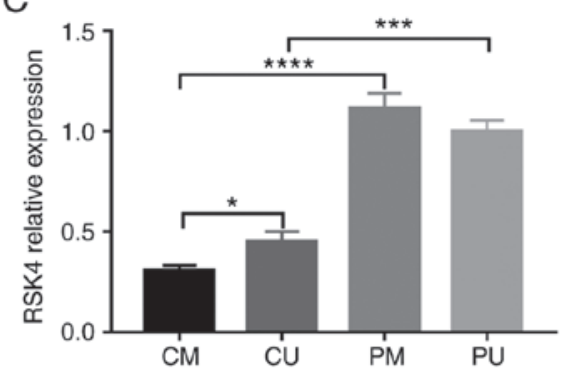

D

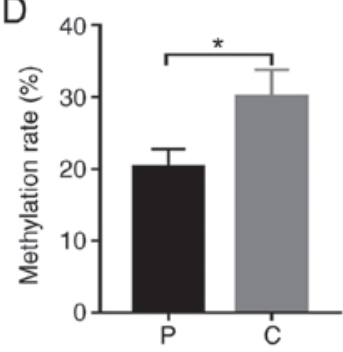

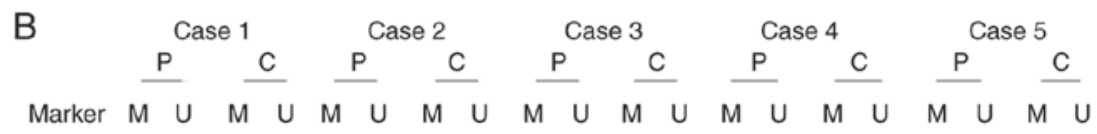
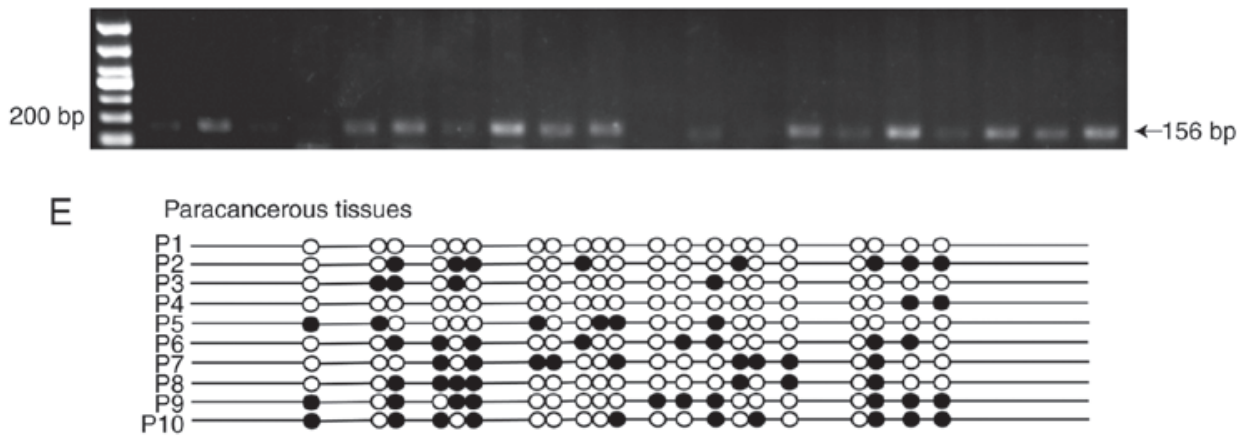

Papillary thyroid carcinoma tissues

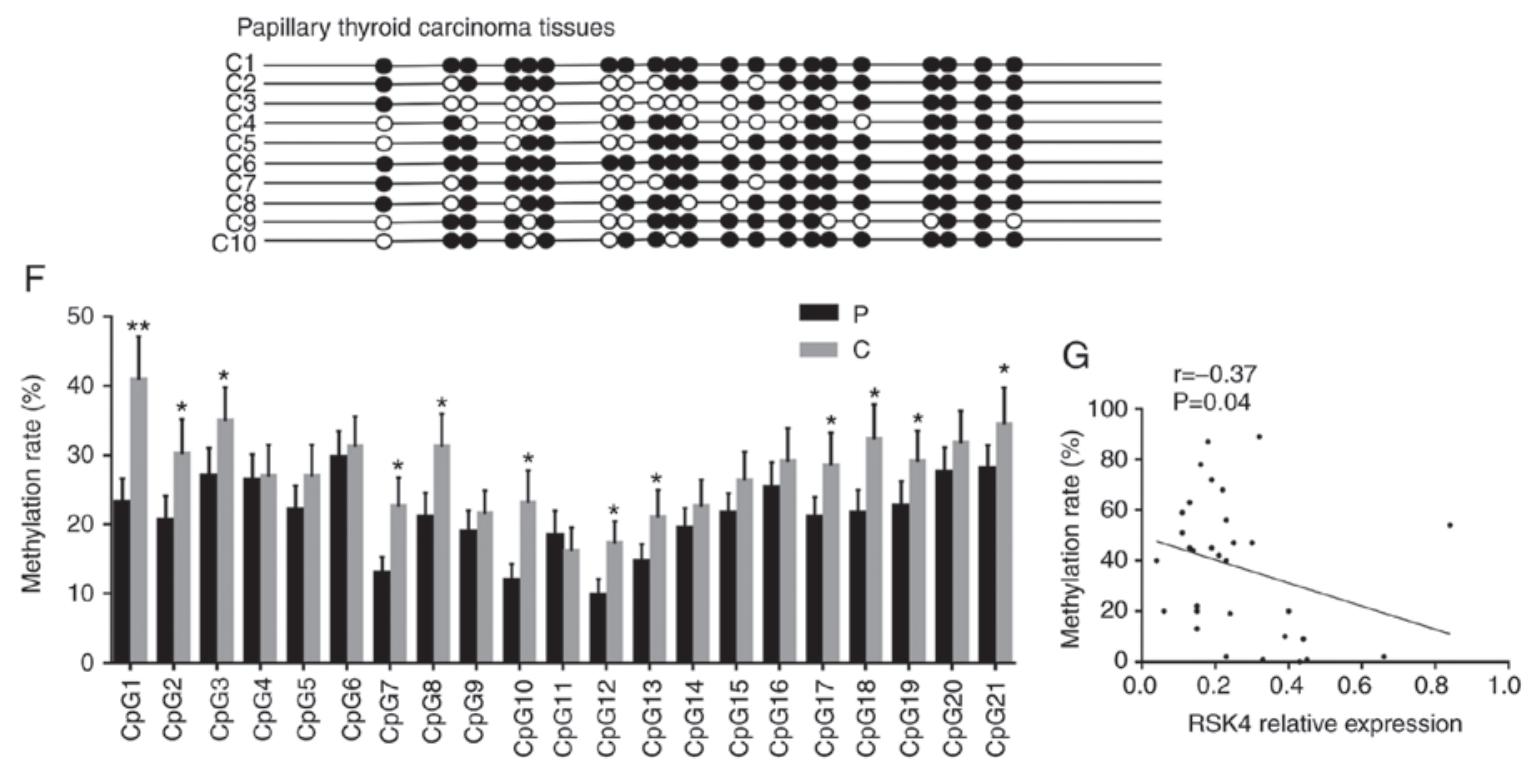

Figure 1. RSK4 is downregulated and its promoter region is hypermethylated in PTC tissues. (A) RSK4 expression in PTC tissues and paired paracancerous tissues, analyzed by RT-qPCR ( $\mathrm{n}=226)$. (B) Representative agarose gel electrophoretogram showing different types of MSP products (P, paracancerous tissues; C, PTC tissues; U, unmethylated; M, methylated). (C) RSK4 expression in different groups, analyzed by RT-qPCR (CU, unmethylated PTC tissues; CM, methylated PTC tissues; PU, unmethylated paracancerous tissues; PM, methylated paracancerous tissues). (D) Methylation rate in PTC tissues and paired paracancerous tissues, analyzed by BGS. (E) BGS analysis of methylation in RSK4 promoter. A total of 21CpG sites of 10 patients were randomly selected from 31 patients analyzed by BGS. The black and white circles indicate the methylated and unmethylated $\mathrm{CpG}$ sites, respectively. (F) Methylation ratio of each CpG sites, identified in the sequenced RSK4 promoter region from PTC and paired paracancerous tissues. (G) Analysis of correlation between RSK4 mRNA expression and the methylation rates in PTC tissues. ${ }^{*} \mathrm{P}<0.05 ;{ }^{* *} \mathrm{P}<0.01 ;{ }^{* * * *} \mathrm{P}<0.001 ;{ }^{* * * *} \mathrm{P}<0.0001$, compared to paracancerous tissues or as indicated. RSK4, ribosomal S6 kinase 4; PTC, papillary thyroid carcinoma; MSP, methylation-specific PCR; BGS, bisulfite genomic sequencing.

and 5'-GACAACCGCTCGATAATAAC-3') were utilized. The modified DNA was amplified by PCR using the following conditions: $98^{\circ} \mathrm{C}$ for $5 \mathrm{~min}$, followed by 35 cycles of $98^{\circ} \mathrm{C}$ for $30 \mathrm{sec}, 56^{\circ} \mathrm{C}$ for $30 \mathrm{sec}$ and $72^{\circ} \mathrm{C}$ for $30 \mathrm{sec}$, and a final 5 -min incubation at $72^{\circ} \mathrm{C}$. $\mathrm{PCR}$ products were isolated by agarose gel electrophoresis.
Bisulfite sequencing (BGS). In total, 31 pairs of specimens of PTC patients were included. Modified DNA samples were subjected to PCR amplification to further analyze the methylation status of the $R S K 4$ promoter. The primers used for bisulfite genomic sequencing were as follows: Forward, 5'-TTTTAA GTTGGGGAATTTTTATTGA-3' and reverse, 5'-AAAACA 
A
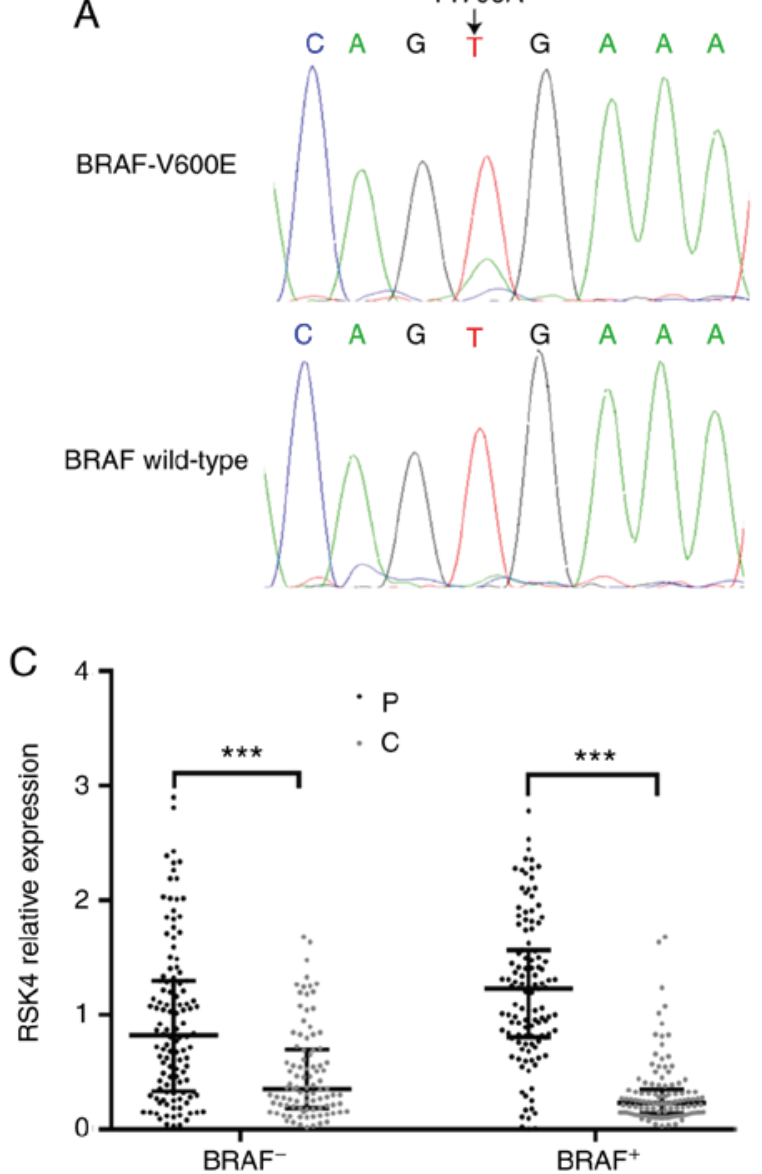
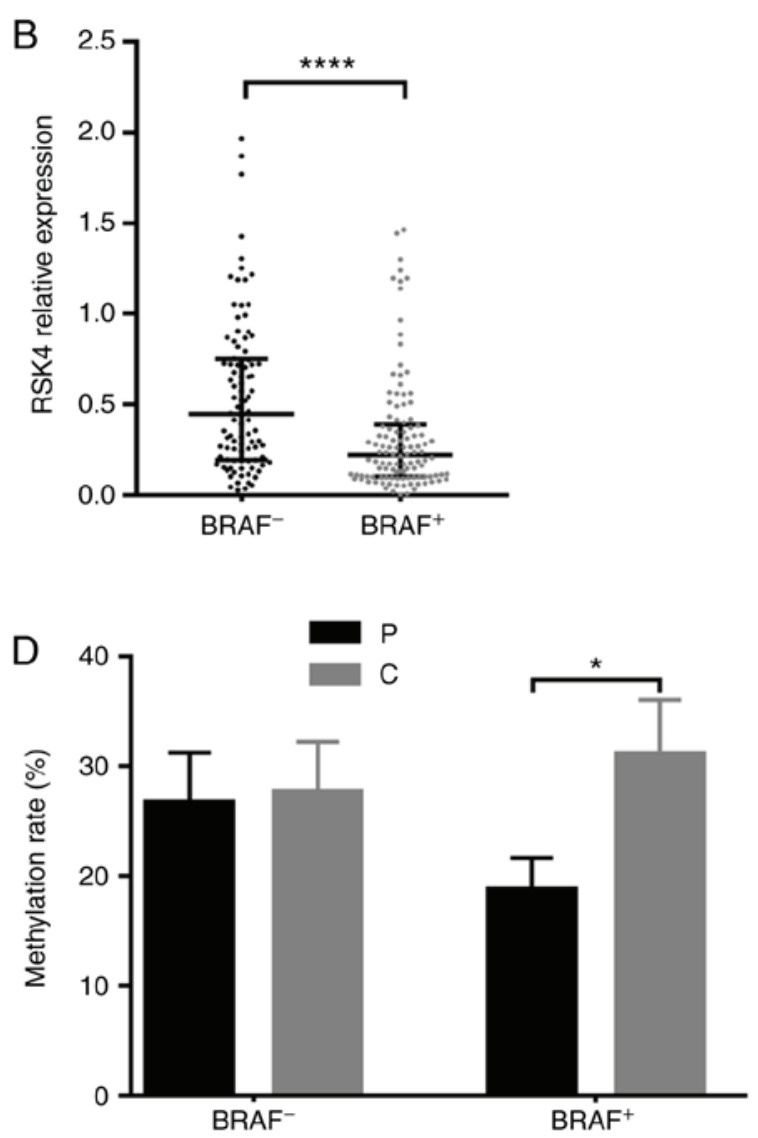

Figure 2. Association between BRAF V600E mutation with RSK4 expression and methylation in PTC tissues. (A) Sequence chromatograms of PTC DNA with non-mutated $B R A F$ and $B R A F V 600 E$ mutation. (B) $R S K 4$ mRNA levels (PTC relative to paracancerous tissues) in patients with non-mutated $B R A F$ (BRAF-) and $B R A F$ V600E mutation $\left(\mathrm{BRAF}^{+}\right)$. (C) RSK4 expression in PTC and paracancerous tissues, grouped by $B R A F$. (D) BGS methylation rate in PTC and paracancerous tissues, grouped by $B R A F$. ${ }^{*} \mathrm{P}<0.05 ;{ }^{* * *} \mathrm{P}<0.001 ;{ }^{* * * *} \mathrm{P}<0.0001$. RSK4, ribosomal S6 kinase 4; PTC, papillary thyroid carcinoma; BGS, bisulfite genomic sequencing.

ACTCTATACTACCTCTCCAAAAAC-3' (21). DNA samples were amplified by PCR using the following conditions: $98^{\circ} \mathrm{C}$ for $5 \mathrm{~min}$, followed by 35 cycles of $98^{\circ} \mathrm{C}$ for $30 \mathrm{sec}, 56^{\circ} \mathrm{C}$ for $30 \mathrm{sec}$ and $72^{\circ} \mathrm{C}$ for $30 \mathrm{sec}$, and a final 5-min incubation at $72^{\circ} \mathrm{C}$. PCR products were ligated into the pMD18-T Vector (Takara Bio, Inc.), followed by transformation into competent the Escherichia coli strain, DH5a (Takara Bio, Inc.). At least 6 clones from each ligation reaction were randomly selected. Bacterial solutions were then sequenced by the Beijing Genomics Institute (China). The percentage of methylated DNA for each sample was computed according to the number of methylated $\mathrm{CpG}$ dinucleotides/(6x21)x100\%.

BRAF V600E mutation analysis. The hot spot region of BRAF V600E mutation was amplified using Premix $\mathrm{Taq}^{\mathrm{TM}}$ (Takara, Code No. R004). The following PCR primers were used: Forward, 5'-GCTTGCTCTGATAGGAAAATG AG-3' and reverse, 5'-GTAACTCAGCAGCATCTCAGG-3'. Genomic DNA was amplified by PCR using following conditions: $95^{\circ} \mathrm{C}$ for $3 \mathrm{~min}$, followed by 38 cycles of $95^{\circ} \mathrm{C}$ for $30 \mathrm{sec}, 57^{\circ} \mathrm{C}$ for $30 \mathrm{sec}$ and $72^{\circ} \mathrm{C}$ for $45 \mathrm{sec}$, and a final 10 -min incubation at $72^{\circ} \mathrm{C}$. Respective PCR products were sequenced by the Beijing Genomics Institute to confirm the $B R A F$ V600E mutation.
Cell transfection. siRNAs targeting BRAF (5'-GCAUCAAUG GAUACCGUUA-3') and control siRNAs (5'-UUCUCCGAA CGUGUCACGU-3') were synthesized by GenPharma. To knockdown BRAF V600E, siRNAs were transfected into the BHT101 cells using HiPerFect Transfection Reagent (Qiagen), according to the manufacturer's protocol. Until transfection, the cells were incubated at $37^{\circ} \mathrm{C}$ and $5 \% \mathrm{CO}_{2}$, and the transfection complexes which had been incubated at room temperature for $10 \mathrm{~min}$ were then added to the cells. After $24 \mathrm{~h}$, the cells were harvested and total RNA was extracted.

Cell proliferation assay. The proliferation of the TPC-1 and BHT101 cells was measured using the cell counting kit-8 (MedChemExpress). For this, cells were seeded into 96-well culture plates $\left(2 \times 10^{3}\right.$ cells/well) and cultured accordingly for $24 \mathrm{~h}$. CCK-8 reagent was added (10 $\mu \mathrm{l} /$ well) at the $0,24,48$, $72,96 \mathrm{~h}$ followed by incubation for an additional $2 \mathrm{~h}$ at $37^{\circ} \mathrm{C}$. The absorbance was measured at $450 \mathrm{~nm}$ using an enzyme immunoassay analyzer (Gen5 software, BioTek Eliasa).

Statistical analysis. Statistical analysis was performed in silico using SPSS (version 23.0. IBM) and Prism 7 (GraphPad Software Inc.). Data with normal distribution are presented as the means \pm SEM. Otherwise, data are expressed as the median 
Table I. Association between the RSK4 methylation rate and clinicopathological features of patients with PTC.

\begin{tabular}{|c|c|c|c|}
\hline Variable & No. of patients & Methylation rate in PTC tissues & P-value \\
\hline \multicolumn{4}{|l|}{ Sex } \\
\hline Male & 16 & $29.86 \pm 4.784$ & \multirow[t]{2}{*}{0.1324} \\
\hline Female & 15 & $18.47 \pm 5.503$ & \\
\hline \multicolumn{4}{|c|}{ Age (years) } \\
\hline$<55$ & 25 & $28.63 \pm 4.134$ & \multirow[t]{2}{*}{0.7537} \\
\hline$\geq 55$ & 6 & $31.75 \pm 10.490$ & \\
\hline \multicolumn{4}{|c|}{ Tumor size $(\mathrm{cm})$} \\
\hline$\leq 1$ & 13 & $26.68 \pm 5.405$ & \multirow[t]{2}{*}{0.5785} \\
\hline$>1$ & 18 & $31.08 \pm 5.392$ & \\
\hline \multicolumn{4}{|c|}{ Lymphatic metastasis } \\
\hline Present & 17 & $37.93 \pm 6.038$ & \multirow[t]{2}{*}{0.0369} \\
\hline Absent & 14 & $22.08 \pm 4.304$ & \\
\hline \multicolumn{4}{|l|}{ Invasion } \\
\hline Present & 8 & $24.01 \pm 8.946$ & \multirow[t]{2}{*}{0.4293} \\
\hline Absent & 23 & $31.06 \pm 4.176$ & \\
\hline \multicolumn{4}{|c|}{ TNM stage } \\
\hline I & 20 & $30.42 \pm 3.795$ & \multirow[t]{2}{*}{0.4296} \\
\hline II & 11 & $21.23 \pm 16.31$ & \\
\hline \multicolumn{4}{|c|}{ Hashimoto thyroiditis } \\
\hline Present & 9 & $31.48 \pm 8.938$ & \multirow[t]{2}{*}{0.8512} \\
\hline Absent & 22 & $29.00 \pm 4.163$ & \\
\hline
\end{tabular}

with the first and third interquartile range (Q1, Q3). Paired samples from the same patients were compared using the paired Student's t-test. An unpaired t-test or two-tailed Mann Whitney U test was used to analyze the difference between 2 groups. Multiple groups ( $\geq 3$ ) were compared using one-way analysis of variance ANOVA and the Bonferroni multiple comparisons test. Spearman's correlation analysis was used to analyze the correlation between $R S K 4$ mRNA expression and the methylation rates in PTC tissues. The difference of rate was analyzed using the Chi-square test. $\mathrm{P}<0.05$ was considered to indicate a statistically significant difference.

\section{Results}

Reduced RSK4 expression and hypermethylation of its promoter region in PTC. To verify whether the inactivation of RSK4 may contribute to thyroid carcinogenesis, RSK4 expression in PTC tissues and paracancerous counterparts was analyzed by RT-qPCR. The $R S K 4 \mathrm{mRNA}$ levels were notably reduced in the PTC tissues when compared with their counterparts $(n=226, P<0.0001)$ (Fig. 1A).

In order to assess the possible mechanism of the downregulation of $R S K 4$ levels in PTC, the methylation status of its gene was examined. A 240-bp region, including $21 \mathrm{CpG}$ sites located in the $R S K 4$ promoter, was analyzed. MSP and BGS analyses were performed to investigate whether a promoter-specific CpG methylation was associated with the decreased RSK4 expression. MSP analysis revealed that the methylation rates of $R S K 4$ in the PTC tissues (93/134, 69.40\%) were higher than those in the paracancerous tissues $(75 / 134$, $55.97 \%, \mathrm{P}<0.05)$. RSK4 expression in methylated PTC tissues $(n=93)$ was reduced compared with that in unmethylated PTC tissues $(n=41, P<0.05)$ (Fig. $1 C)$, with no significant difference in expression in the paracancerous tissues $(\mathrm{P}>0.05)$. RSK4 expression in methylated PTC tissues was lower than that in methylated paracancerous tissues $(\mathrm{n}=74, \mathrm{P}<0.0001)$. Moreover, RSK4 expression in unmethylated PTC tissues was also lower than that in unmethylated paracancerous tissues $(n=60$, $\mathrm{P}<0.001$ ) (Fig. 1C).

BGS analysis also provided evidence that the methylation rates were higher in $\mathrm{PTC}$ tissues $(\mathrm{n}=31, \mathrm{P}<0.05)$ (Fig. $1 \mathrm{D}$ and $\mathrm{E})$. In the majority of $\mathrm{CpG}$ sites, the methylation rates of the $R S K 4$ promoter were higher in PTC than in paracancerous tissues (Fig. 1F). In addition, it was found that RSK4 mRNA expression negatively correlated with the methylation rates in PTC tissues ( $\mathrm{r}=-0.37, \mathrm{P}<0.05)$ (Fig. 1G). Taken together, these results indicate that the methylation rates of the $R S K 4$ promoter are increased in PTC, leading to the downregulation of $R S K 4$ expression.

Association between BRAF V600E mutation, and the expression and methylation of RSK4 in PTC. Previous studies have revealed that RSK4 is involved in MAPK signaling transduction and, therefore, it can regulate ERK activation. Thus, the present study investigated whether there was any association between the BRAF V600E mutation with RSK4 expression and methylation in PTC. The chromatograms of DNA sequencing are presented in Fig. 2A, denoting BRAF T1799A and 
Table II. Association between the RSK4 methylation status and clinicopathological features of patients with PTC with or without BRAF V600E mutation.

\begin{tabular}{|c|c|c|c|c|c|c|c|c|}
\hline \multirow[b]{2}{*}{ Variable } & \multicolumn{4}{|c|}{$\mathrm{BRAF}^{+}$} & \multicolumn{4}{|c|}{$\mathrm{BRAF}^{-}$} \\
\hline & $\begin{array}{l}\text { No. of } \\
\text { patients }\end{array}$ & Methylated & Unmethylated & P-value & $\begin{array}{c}\text { No. of } \\
\text { patients }\end{array}$ & Methylated & Unmethylated & P-value \\
\hline \multicolumn{9}{|l|}{ Sex } \\
\hline Male & 23 & 15 & 8 & 0.2906 & 12 & 5 & 7 & 0.0586 \\
\hline Female & 48 & 37 & 11 & & 51 & 36 & 15 & \\
\hline \multicolumn{9}{|c|}{ Age (years) } \\
\hline$<55$ & 60 & 44 & 16 & 0.9667 & 55 & 36 & 19 & 0.8699 \\
\hline$\geq 55$ & 11 & 8 & 3 & & 8 & 5 & 3 & \\
\hline \multicolumn{9}{|c|}{ Tumor size (cm) } \\
\hline$\leq 1$ & 30 & 18 & 12 & 0.0311 & 36 & 22 & 14 & 0.4455 \\
\hline$>1$ & 41 & 34 & 7 & & 27 & 19 & 8 & \\
\hline \multicolumn{9}{|c|}{ Lymphatic metastasis } \\
\hline Present & 44 & 36 & 8 & 0.0371 & 31 & 22 & 9 & 0.3346 \\
\hline Absent & 27 & 16 & 11 & & 32 & 19 & 13 & \\
\hline \multicolumn{9}{|l|}{ Invasion } \\
\hline Present & 16 & 10 & 6 & 0.2703 & 9 & 4 & 5 & 0.1607 \\
\hline Absent & 55 & 42 & 13 & & 54 & 37 & 17 & \\
\hline \multicolumn{9}{|c|}{ TNM stage } \\
\hline I & 58 & 42 & 16 & 0.7399 & 55 & 36 & 19 & 0.8699 \\
\hline II & 13 & 10 & 3 & & 8 & 5 & 3 & \\
\hline \multicolumn{9}{|c|}{ Hashimoto thyroiditis } \\
\hline Present & 10 & 6 & 4 & 0.3076 & 19 & 11 & 8 & 0.4318 \\
\hline Absent & 61 & 46 & 15 & & 44 & 30 & 14 & \\
\hline
\end{tabular}

wild-type $B R A F$. It was found that the $R S K 4$ mRNA levels (PTC relative to the paracancerous tissues) in PTC patients with $B R A F$ V600E mutation $(\mathrm{n}=124)$ were significantly lower than those with wild-type $B R A F$ (n=102, $\mathrm{P}<0.0001$, Fig. 2B). Independently of the $B R A F$ mutation status, the RSK4 mRNA levels in PTC tissues were lower than those in paracancerous tissues (Fig. 2C). However, only in patients with BRAF V600E mutation, the methylated rates in the PTC tissues were higher than those in paracancerous tissues, as detected by MSP (73.23 vs. 50.7\%, $\mathrm{P}<0.001)$ and BGS $(\mathrm{P}<0.05)$ (Fig. 2D). Taken together, these findings indicate that $B R A F$ V600E mutation may be one of reasons for the downregulation of $R S K 4$ mRNA levels due to the increasing methylation rates in PTC.

Association between RSK4 methylation levels and the clinicopathological characteristics of patients with PTC. To further explore the role of $R S K 4$ hypermethylation in PTC, 134 (MSP) and 31 (BGS) PTC patients with available information were analyzed. BGS analysis revealed that the methylation rates were higher in tumors with lymph node metastasis (Table I). MSP analysis revealed that the methylation rates in PTC tumors $>1.0 \mathrm{~cm}$ in diameter $(34 / 41$, $82.93 \%$ ) were higher than those in PTC tumors $\leq 1.0 \mathrm{~cm}$ in diameter $(18 / 30,60 \%)$ in patients with $B R A F$ V600E mutation (Table II). In addition, the methylation rates were higher in tumors with lymph node metastasis $(36 / 44,81.82 \%)$ compared with those without metastasis $(16 / 27,59.26 \%)$ in patients with $B R A F$ V600E mutation (Table II). PTC patients with Hashimoto thyroiditis were also included to examine whether there was an association between the methylation status of RSK4 and Hashimoto thyroiditis in PTC; it was found that the methylation rate of $R S K 4$ was not related to Hashimoto thyroiditis (Tables I and II). These findings are in accordance with a tumor suppressor role for RSK4 in inhibiting tumor growth and metastasis in PTC.

RSK4 expression associated with BRAF V600E is downregulated and hypermethylated in thyroid cancer cells. RSK4 levels and the methylation status of its promoter were examined in vitro, using a normal (Nthy-ori3-1) and thyroid cancer cell lines (TPC-1 and BHT101). The results revealed higher methylation rates of the RSK4 promoter in TPC-1 and BHT101 when compared with Nthy-ori3-1 cells (Fig. 3C). By contrast, both the RSK4 mRNA and protein levels were lower in the TPC-1 and BHT101 cells when compared with the Nthy-ori3-1 cells (Fig. 3A and B). As shown in Fig. 3D and E, $B R A F$ expression was significantly downregulated and RSK4 expression was significantly upregulated following the knockdown of BRAF V600E in the BHT101 cells. These findings suggest that RSK4 expression is negatively associated with the methylation status of its promoter and is also influenced by BRAF V600E in thyroid cancer cells. These in vitro data 

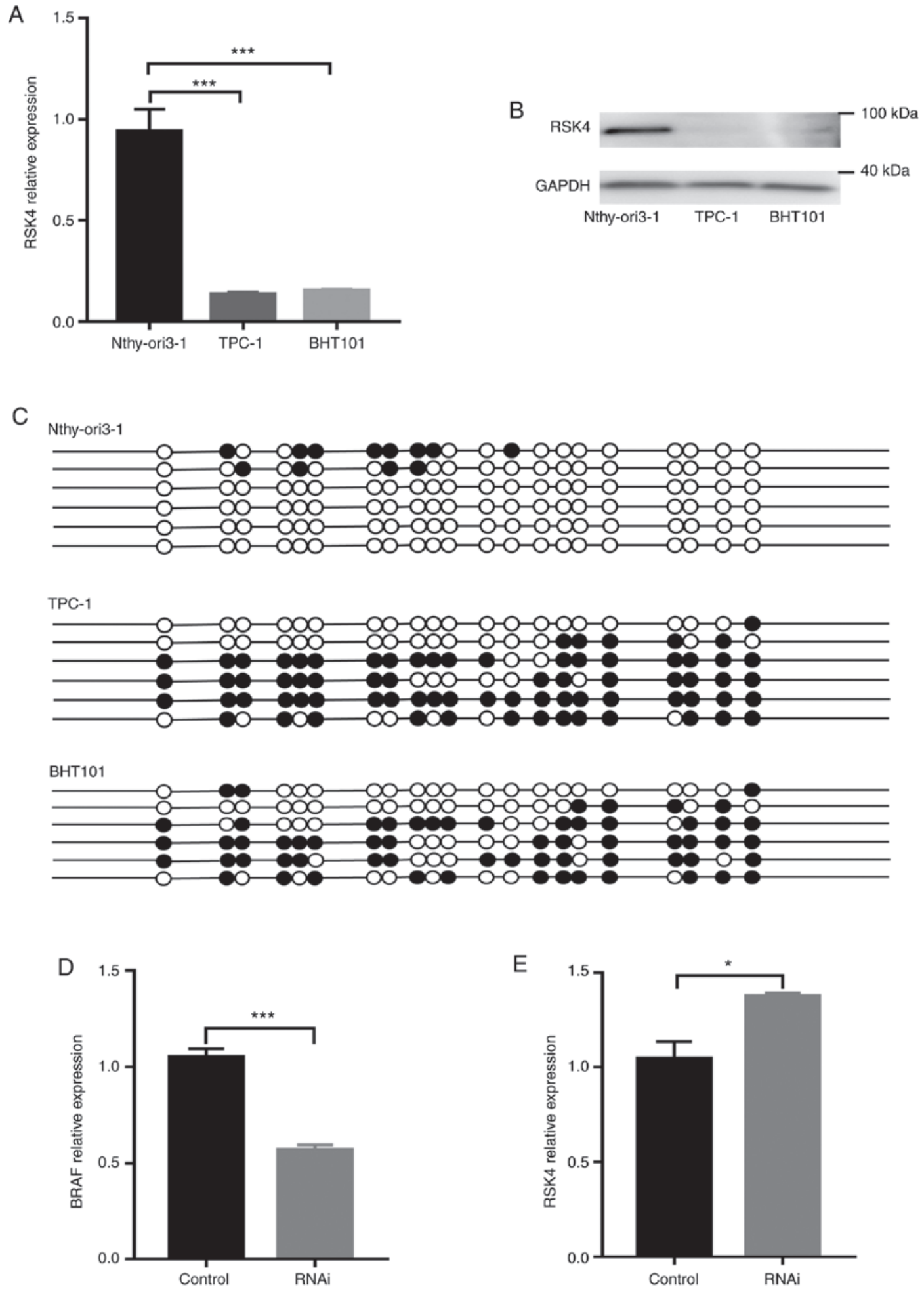

Figure 3. RSK4 expression and methylation status in Nthy-ori3-1, TPC-1 and BHT101 cell lines. (A) RSK4 mRNA levels in Nthy-ori3-1, TPC-1 and BHT101, detected by RT-qPCR. (B) RSK4 protein levels in Nthy-ori3-1, TPC-1 and BHT101 cells, detected by western blot analysis. GAPDH was used as an internal control. (C) BGS analysis of methylation in RSK4 promoter from 21 CpG sites of 6 clones from Nthy-ori3-1, TPC-1 and BHT101 cells. The black and white circles indicate the methylated and unmethylated sites. (D and E) BRAF and RSK4 expression in BHT101 cells, detected by RT-qPCR following transfection with siRNA targeting BRAF. ${ }^{*} \mathrm{P}<0.05 ;{ }^{* * *} \mathrm{P}<0.001$. RSK4, ribosomal S6 kinase 4; BGS, bisulfite genomic sequencing.

are consistent with the results obtained with the human tissue specimens mentioned above.

RSK4 expression is enhanced by 5-Aza and DNA demethylation inhibits the proliferation of thyroid cancer cells. To further investigate the role of promoter hypermethylation in the regulation of RSK4 expression, the TPC-1, BHT101 and Nthy-ori3-1 cells were treated with the demethylating agent, 5-Aza. The results revealed that the RSK4 mRNA and protein levels were restored in the TPC-1 and BHT101 cells, but not in the Nthy-ori3-1 cells (Fig. 4A and B). Furthermore, the proliferative ability of the TPC-1 and BHT101 cells was significantly 

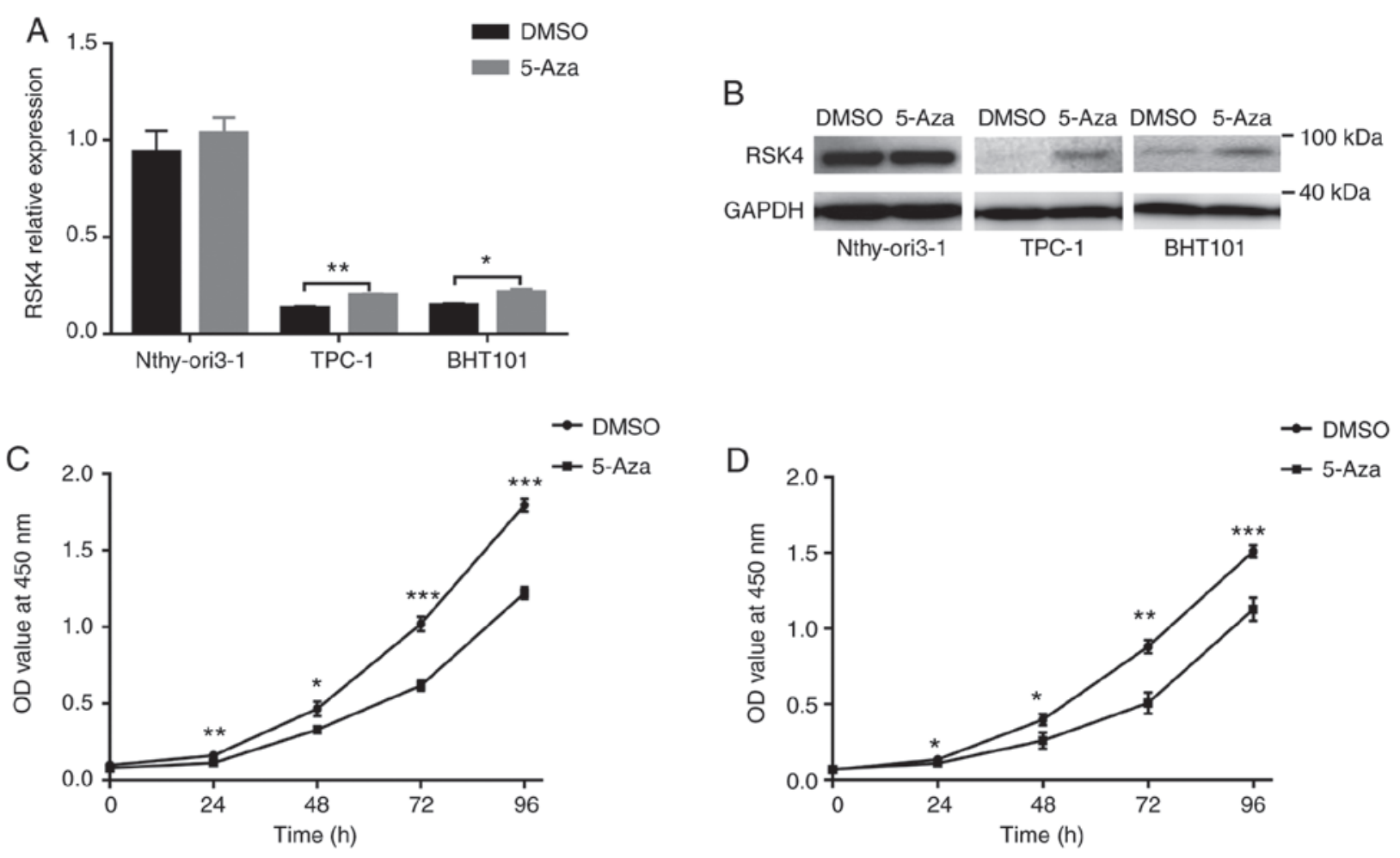

Figure 4. RSK4 expression and cell proliferation upon treatment with the demethylating agent, 5-Aza. (A and B) RSK4 mRNA and protein levels, analyzed by RT-qPCR and western blot analysis in Nthy-ori3-1, TPC-1 and BHT101 cells treated (or not) with $10 \mu \mathrm{M}$ 5-Aza. DMSO treatment was performed as a negative control. Proliferation of (C) TPC-1 and (D) BHT101 cells, determined by CCK-8 assay following treatment with $10 \mu$ M 5-Aza (using DMSO as a control). ${ }^{*} \mathrm{P}<0.05 ;{ }^{* *} \mathrm{P}<0.01 ;{ }^{* * *} \mathrm{P}<0.001$. RSK4, ribosomal S6 kinase 4; 5-Aza, 5-Aza-deoxycytidine.

suppressed by treatment with $10 \mu \mathrm{M}$ 5-Aza (Fig. 4C and D). These data suggest that the hypermethylation of the RSK4 promoter can reduce gene expression and, importantly, DNA demethylation can lead to the repression of thyroid cancerous cell proliferation.

\section{Discussion}

Papillary thyroid cancer (PTC) accounts for $80-90 \%$ of all thyroid cancers and is the most common endocrine malignancy, with a rapidly increasing incidence worldwide (22). Although thyroid cancer has been frequently associated with a relatively indolent progress and a good prognosis, some patients become incurable with notable morbidity and mortality $(3,23)$. In spite of the overall 10 -year survival rate (approximately 90\%), approximately $10-20 \%$ of patients with PTC with stage I or II disease suffer from disease recurrence (24). The molecular pathogenesis of thyroid cancer involves genetic alterations of multiple oncogenes, such as $B R A F, T E R T$ and $R A S$, and tumor suppressor genes, such as PTEN and RASAL1. Their potential association with clinicopathologic aggressiveness and the poor prognosis of thyroid cancer has been partially demonstrated $(12,14,25)$. Advances in the molecular pathogenesis of thyroid cancer have exhibited great prospect toward more effective diagnosis and treatment strategies for this disease (12). Moreover, the understanding of these genetic alterations may further lead to a reduction in unnecessary treatments for indolent PTC, and may also improve the prognosis of patients affected by clinically aggressive cancers (3).
RSK4 is a functional ribosome protein kinase that belongs to the p90 RSK family. Recent studies have demonstrated its apparent significance in tumorigenesis, since a low or no RSK4 expression has been associated with malignant tumors of the colon, kidney, ovary, pancreas, hematological system and endometrium $(8,9,26-28)$. Specifically, it has been demonstrated that RSK4 may drive cellular senescence by modulating $\mathrm{Rb}$ and $\mathrm{p} 21$, since the upregulation of RSK4 expression can increase p21 levels $(6,7,29)$. These studies have indicated that the downregulation of RSK4 may be an important element in promoting cell transformation and, therefore, may support the notion of the role of RSK4 in regulating senescence as tumor suppressor protein $(6-8,16)$. Similarly, the present study found that RSK4 expression was significantly decreased in the majority of PTC tissues and in a PTC-derived cell line.

Promoter hypermethylation has been recognized as standard mechanism that leads to the transcriptional inactivation of tumor suppressor genes (TSGS) in a variety of cancer types (30). Some studies have indicated that the hypermethylation of the RSK4 promoter can lead to a decrease in its own expression in several malignancies, including breast, endometrial, oral and ovarian cancers. Accordingly, the decreased RSK4 expression has been restored by DNA methyltransferase inhibitors in several cancer cell lines $(21,27,30,31)$. However, no studies to date have reported the methylation status of RSK4 in PTC and paracancerous tissues, at least to the best of our knowledge. Hence, the authors conducted MSP and BGS to finally examine the methylation status of RSK4. Both approaches were able to demonstrate that the methylation 
rates of $R S K 4$ promoter were consistently higher in PTC than in paracancerous tissues. This increased methylation status negatively correlated with RSK4 expression in PTC tissues. Upon methylation, the RSK4 mRNA levels in PTC were lower than those in paracancerous tissues. Under non-methylating conditions, the RSK4 mRNA levels were less prominently decreased in PTC when compared with those in paracancerous tissues. As stated, it appears that RSK4 methylation may coexist in both PTC and paracancerous tissues, where promoter methylation may be one of the various mechanisms that can lead to a reduction in RSK4 expression. According to the clinicopathological data, this study also demonstrated the association between $R S K 4$ promoter methylation with lymph node metastasis and tumor size, providing evidence that $R S K 4$ methylation may play a role in the development and prognosis of PTC. Indeed, previous studies have indicated that RSK4 can suppress tumor growth, invasiveness and metastatic ability of other cancer cell lines $(7,8)$.

Strikingly, the aberrant RSK4 expression in PTC cells could be reversed by broad-spectrum demethylated drugs, and DNA demethylation inhibited the proliferation of thyroid cancer cells. In other words, the tumor suppressor effect of $R S K 4$, to a certain extent, could be restored by demethylation, suggesting that targeted demethylation drugs may be an attractive option for tumor therapy in the near future.

An extensive number of genetic alterations have been involved in the tumorigenesis of various thyroid cancers. The T1799A transverse point mutation of $B R A F$ is a prototypical example, which leads to the expression of a $B R A F V 600 E$ mutant protein and contributes to constitutive activation of this serine/threonine kinase. BRAF V600E mutation can activate the MAPK signaling pathway, which is a key feature of PTC (12,32). A previous comprehensive and multicenter study demonstrated a strong association between $B R A F$ V600E with the poor clinicopathological outcomes of PTC, including aggressive pathological characteristics, disease recurrence, loss of radioiodine avidity and unfavorable prognosis (23,33-35). Previous studies have demonstrated that RSK4 can negatively modulate the MAPK pathway at the ERK or the downstream levels, depending on its kinase activity $(10,26)$. The present study analyzed the status of $B R A F$ mutations in $226 \mathrm{PTC}$ tissues and, as a result, it was found that BRAF V600E mutation rates were considerably high. Furthermore, this study indicates that BRAF V600E mutation may influence RSK4 expression, leading to a more significant difference on mRNA levels between PTC tissues and paracancerous tissues. Moreover, it was found that this mutation can influence the methylation status of $R S K 4$, thereby influencing the clinicopathological outcomes of patients with PTC, which reiterates the putative role of $R S K 4$ as a cancer suppressor gene in thyroid tumors.

To conclude, the present study demonstrates that the hypermethylation of $\mathrm{CpG}$ islands is one prominent mechanism that can lead the downregulation of RSK4 expression. The hypermethylation of the $R S K 4$ promoter is associated with tumor proliferation and lymph node metastasis. $B R A F$ V600E mutation also has an effect on RSK4 mRNA levels and gene methylation status, supporting the distinctive role of $R S K 4$ in the development, progression and prognosis of PTC.

\section{Acknowledgements}

The authors would like to thank all of the investigators for their involvement in this study.

\section{Funding}

The present study was supported by the Key research and Development program of Shandong Province of China (2018GSF118051); the China Postdoctoral Science Foundation Grant (2019M662303); the Natural Science Foundation of Shandong Province of China (ZR2016HM29); and the Funded program of Science and Technology Development of Shinan District, Qingdao, Shandong Province of China (2018-4-013-YY).

\section{Availability of data and materials}

The data that support the findings of this study are available from the corresponding author upon reasonable request.

\section{Authors' contributions}

YY, YW, JC and WS designed the study and wrote the manuscript. YY, JC, KC and WS performed the majority of the experiments. YW, JH, HH, AD, JW, QZ, JY, SZ, YZ, PW and $F W$ participated in the experimentation. WS provided the patients and participated in the discussions. All authors have read and approved the final manuscript.

\section{Ethics approval and consent to participate}

The study protocol was approved by the Ethics Committee of Affiliated Hospital of Qingdao University (QDFY WZ 2018-1-14-02) and was conducted in accordance with the Helsinki Declaration of 1964 and its later amendments or comparable ethical standards. All patients provided written informed consent. For patients $<18$ years old, their written informed consents were provided by their parents.

\section{Patient consent for publication}

Not applicable.

\section{Competing interests}

All authors declare that they have no competing interests.

\section{References}

1. Torre LA, Bray F, Siegel RL, Ferlay J, Lortet-Tieulent J and Jemal A: Global cancer statistics, 2012. CA Cancer J Clin 65: 87-108, 2015.

2. Haugen BR, Alexander EK, Bible KC, Doherty GM, Mandel SJ, Nikiforov YE, Pacini F, Randolph GW, Sawka AM, Schlumberger M, et al: 2015 American thyroid association management guidelines for adult patients with thyroid nodules and differentiated thyroid cancer: The American thyroid association guidelines task force on thyroid nodules and differentiated thyroid cancer. Thyroid 26: 1-133, 2016.

3. Cabanillas ME, McFadden DG and Durante C: Thyroid cancer. Lancet 388: 2783-2795, 2016.

4. Tuttle RM, Ball DW, Byrd D, Dilawari RA, Doherty GM, Duh QY, Ehya H, Farrar WB, Haddad RI, Kandeel F, et al: Thyroid carcinoma. J Natl Compr Canc Netw 8: 1228-1274, 2010. 
5. Riesco-Eizaguirre G and Santisteban P: ENDOCRINE TUMOURS: Advances in the molecular pathogenesis of thyroid cancer: Lessons from the cancer genome. Eur J Endocrinol 175: R203-R217, 2016

6. López-Vicente L, Pons B, Coch L, Teixidó C, Hernández-Losa J, Armengol G and Ramon Y Cajal S: RSK4 inhibition results in bypass of stress-induced and oncogene-induced senescence. Carcinogenesis 32: 470-476, 2011.

7. López-Vicente L, Armengol G, Pons B, Coch L, Argelaguet E, Lleonart M, Hernández-Losa J, de Torres I and Ramon y Cajal S: Regulation of replicative and stress-induced senescence by RSK4, which is down-regulated in human tumors. Clin Cancer Res 15: 4546-4553, 2009.

8. Arechavaleta-Velasco F,Zeferino-Toquero M,Estrada-Moscoso I, Imani-Razavi FS, Olivares A, Perez-Juarez CE and Diaz-Cueto L: Ribosomal S6 kinase 4 (RSK4) expression in ovarian tumors and its regulation by antineoplastic drugs in ovarian cancer cell lines. Med Oncol 33: 11, 2016.

9. Dewdney SB, Rimel BJ, Thaker PH, Thompson DM Jr, Schmidt A, Huettner P, Mutch DG, Gao F and Goodfellow PJ: Aberrant methylation of the X-linked ribosomal S6 kinase RPS6KA6 (RSK4) in endometrial cancers. Clin Cancer Res 17: 2120-2129, 2011

10. Jiang Y, Ye X, Ji Y, Zhou X, Yang H, Wei W and Li Q: Aberrant expression of RSK4 in breast cancer and its role in the regulation of tumorigenicity. Int J Mol Med 40: 883-890, 2017.

11. Anjum R and Blenis J: The RSK family of kinases: Emerging roles in cellular signalling. Nat Rev Mol Cell Biol 9: 747-758, 2008.

12. Xing M: Molecular pathogenesis and mechanisms of thyroid cancer. Nat Rev Cancer 13: 184-199, 2013.

13. Lin JD, Fu SS, Chen JY, Lee CH, Chau WK, Cheng CW, Wang YH, Lin YF, Fang WF and Tang KT: Clinical manifestations and gene expression in patients with conventional papillary thyroid carcinoma carrying the BRAF(V600E) mutation and BRAF pseudogene. Thyroid 26: 691-704, 2016.

14. Xing M: Genetic-guided risk assessment and management of thyroid cancer. Endocrinol Metab Clin North Am 48: 109-124, 2019.

15. Yang T, Chen C, Pan NF, Sun LY, Jiang XL, Li JN, Tang Y and Jiang Y: BRAF V600E mutation and TERT promoter mutation in papillary thyroid carcinomas and their association with clinicopathological characteristics. Sichuan Da Xue Xue Bao Yi Xue Ban 50: 919-924, 2019 (In Chinese)

16. Myers AP, Corson LB, Rossant J and Baker JC: Characterization of mouse Rsk4 as an inhibitor of fibroblast growth factor-RAS-extracellular signal-regulated kinase signaling. Mol Cell Biol 24: 4255-4266, 2004.

17. Peixoto P, Grandvallet C, Feugeas JP, Guittaut M and Hervouet E: Epigenetic control of autophagy in cancer cells: A key process for cancer-related phenotypes. Cells 8: E1656, 2019.

18. Skvortsova K, Stirzaker C and Taberlay P: The DNA methylation landscape in cancer. Essays Biochem 63: 797-811, 2019.

19. Curtis CD and Goggins M: DNA methylation analysis in human cancer. Methods Mol Med 103: 123-136, 2005.

20. Kubista M, Andrade JM, Bengtsson M, Forootan A, Jonák J, Lind K, Sindelka R, Sjöback R, Sjögreen B, Strömbom L, et al: The real-time polymerase chain reaction. Mol Aspects Med 27: 95-125, 2006.

21. Niskakoski A, Kaur S, Staff S, Renkonen-Sinisalo L, Lassus H, Järvinen HJ, Mecklin JP, Bützow R and Peltomäki P: Epigenetic analysis of sporadic and Lynch-associated ovarian cancers reveals histology-specific patterns of DNA methylation. Epigenetics 9: $1577-1587,2014$
22. Mao Y and Xing M: Recent incidences and differential trends of thyroid cancer in the USA. Endocr Relat Cancer 23: 313-322, 2016.

23. Liu R, Bishop J, Zhu G, Zhang T, Ladenson PW and Xing M: Mortality risk stratification by combining BRAF V600E and TERT promoter mutations in papillary thyroid cancer: Genetic duet of BRAF and TERT promoter mutations in thyroid cancer mortality. JAMA Oncol 3: 202-208, 2017.

24. Tsumagari K, Abd Elmageed ZY, Sholl AB, Friedlander P, Abdraboh M, Xing M, Boulares AH and Kandil E: Simultaneous suppression of the MAP kinase and NF- $\mathrm{KB}$ pathways provides a robust therapeutic potential for thyroid cancer. Cancer Lett 368: $46-53,2015$

25. Liu D, Yang C, Bojdani E, Murugan AK and Xing M: Identification of RASAL1 as a major tumor suppressor gene in thyroid cancer. J Natl Cancer Inst 105: 1617-1627, 2013.

26. Khalaileh A, Dreazen A, Khatib A, Apel R, Swisa A, Kidess-Bassir N, Maitra A, Meyuhas O, Dor Y and Zamir G: Phosphorylation of ribosomal protein S6 attenuates DNA damage and tumor suppression during development of pancreatic cancer. Cancer Res 73: 1811-1820, 2013.

27. Li Q, Jiang Y, Wei W, Ji Y, Gao H and Liu J: Frequent epigenetic inactivation of RSK4 by promoter methylation in cancerous and non-cancerous tissues of breast cancer. Med Oncol 31: 793 , 2014.

28. Rafiee M, Keramati MR, Ayatollahi H, Sadeghian MH, Barzegar M, Asgharzadeh A and Alinejad M: Down-regulation of ribosomal S6 kinase RPS6KA6 in acute myeloid leukemia patients. Cell J 18: 159-164, 2016.

29. LLeonart ME, Vidal F, Gallardo D, Diaz-Fuertes M, Rojo F, Cuatrecasas M, López-Vicente L, Kondoh H, Blanco C, Carnero A, et al: New p53 related genes in human tumors: Significant downregulation in colon and lung carcinomas. Oncol Rep 16: 603-608, 2006

30. Xiao K, Yu Z, Shi DT, Lei Z, Chen H, Cao J, Tian W, Chen W and Zhang HT: Inactivation of BLU is associated with methylation of Sp1-binding site of BLU promoter in gastric cancer. Int J Oncol 47: 621-631, 2015.

31. Foy JP, Pickering CR, Papadimitrakopoulou VA, Jelinek J, Lin SH, William WN Jr, Frederick MJ, Wang J, Lang W, Feng L, et al: New DNA methylation markers and global DNA hypomethylation are associated with oral cancer development. Cancer Prev Res (Phila) 8: 1027-1035, 2015.

32. Xing M, Alzahrani AS, Carson KA, Shong YK, Kim TY, Viola D, Elisei R, Bendlová B, Yip L, Mian C, et al: Association between BRAF V600E mutation and recurrence of papillary thyroid cancer. J Clin Oncol 33: 42-50, 2015.

33. Xing M, Westra WH, Tufano RP, Cohen Y, Rosenbaum E, Rhoden KJ, Carson KA, Vasko V, Larin A, Tallini G, et al: BRAF mutation predicts a poorer clinical prognosis for papillary thyroid cancer. J Clin Endocrinol Metab 90: 6373-6379, 2005.

34. Wang F, Zhao S, Shen X, Zhu G, Liu R, Viola D, Elisei R, Puxeddu E, Fugazzola L, Colombo C, et al: BRAF V600E confers male sex disease-specific mortality risk in patients with papillary thyroid cancer. J Clin Oncol 36: 2787-2795, 2018.

35. Kimura ET, Nikiforova MN, Zhu Z, Knauf JA, Nikiforov YE and Fagin JA: High prevalence of BRAF mutations in thyroid cancer: Genetic evidence for constitutive activation of the RET/PTC-RAS-BRAF signaling pathway in papillary thyroid carcinoma. Cancer Res 63: 1454-1457, 2003. 